\title{
Production analysis for fractured vertical well in rectangular coal reservoirs
}

Chen $\mathrm{Li}^{1}$, Zhenzhen Dong ${ }^{2}{ }^{*}$, and Xiang $\mathrm{Li}^{1}$

${ }^{1}$ College of Engineering, Peking University, Room 1010, WangKeZhen Building, Peking University, NO. 5 Yihe Road, Haidian District, Beijing, PR China

${ }^{2}$ Petroleum Engineering Department, Xi'an Shiyou University, Room 212, 18 Dianzierlu, Yanta District, Xi'an, Shannxi Province, PR China

Received: 15 May 2018 / Accepted: 28 August 2018

\begin{abstract}
As one kind of unconventional natural gas, coalbed methane is an important energy resource that is subject to active research. Gas exists in coalbed methane reservoirs in two forms: free gas and adsorbed gas. In the course of coalbed methane production, the reservoir experiences pressure decrease, desorption, diffusion, and seepage. Previous models of coalbed methane production were mainly concerned with circular boundaries. However, field tests revealed that some fractured wells possess the characteristics of rectangular boundaries. For fractured rectangular coalbed methane reservoirs, it is necessary to deal with the four boundaries with mirror image theory, which complicates calculations. In addition, the desorption and adsorption process of coalbed methane exerts a strong effect on the seepage process. Furthermore, the complexity of the rectangular coal seam embedded with the finite-conductivity fracture results in a significant computational challenge. For the first time, this paper presented a fast analytical solution for a finite-conductivity fractured vertical well model with either rectangular closed or constant-pressure boundaries in the coal seam. On the basis of the Fick diffusion law and the Darcy seepage law, a mathematical model that considers diffusion in matrix and seepage within natural fractures was established. Then, we integrated the fracture conductivity function method with the hydraulic fracture model to greatly increase computational efficiency. The analytical solutions were validated against a numerical simulation. Parameter sensitivity analysis reveals that interporosity coefficient and storage coefficient, respectively, affect the appearance time and degree of desorption and diffusion. Desorption coefficient mainly describes the capacity of desorption and diffusion. Well storage coefficient, conductivity factor, and skin factor mainly affect the early stage of production. Finally, the proposed solutions were applied to field history match. The model developed is applicable to production analysis and well testing for coalbed methane reservoirs. The new proposed model extended flow mechanism of coalbed methane, and provided a better production and pressure forecast for coalbed methane reservoirs. In addition, the analytical solutions can be used to generate type curves for fractured vertical wells with finite conductivity and in the rectangular boundary, and provide a sound theoretical basis for well tests in the coal seam. The model is also applicable to other types of unconventional gas reservoirs, such as gas shales, in which the same processes are present.
\end{abstract}

\section{Nomenclature}

\section{Symbols}

$r \quad$ Radial radius of gas reservoir, $\mathrm{m}$

$\psi \quad$ Pseudo pressure, $\mathrm{MPa}^{2} / \mathrm{cp}$

$p \quad$ Pressure, $\mathrm{MPa}$

$\mu \quad$ Gas viscosity, $\mathrm{mPa} \mathrm{s}$

$Z \quad$ Gas deviation factor, dimensionless

$\phi \quad$ Porosity, decimal $c_{\mathrm{g}} \quad$ Compressibility, $\mathrm{MPa}$

$k \quad$ Permeability, D

$T \quad$ Temperature, $\mathrm{K} \quad-1$

$V \quad$ Gas concentration, $\mathrm{m}^{3} / \mathrm{m}^{3}$

C Volumetric gas concentration in microspores, $\mathrm{m}^{3} / \mathrm{m}^{3}$

D Diffusion coefficient, $\mathrm{m}^{3} / \mathrm{s}$

$p_{\mathrm{L}} \quad$ Langmuir pressure, $\mathrm{MPa}$

$s \quad$ Image variable of Laplace transformation

$R \quad$ External radius of matrix, $\mathrm{m}$ 
$L_{\mathrm{f}} \quad$ Fracture half length, m

DPP Dimensionless pseudo pressure

DPPD Dimensionless pseudo pressure derivative

\section{Subscripts and superscripts}

D Dimensionless property

e Boundary property

w Wellbore property

i Initial condition

sc Standard condition

f Fracture property

Image function of Laplace transform

\section{Intermediate variables}

$\begin{array}{ll}\sigma & \frac{p_{\mathrm{L}} V_{\mathrm{L}} p_{i}^{2} q_{\mathrm{D}}}{\left(p_{\mathrm{L}}+p\right)+\left(p_{\mathrm{L}}+p_{i}\right)+\left(p_{i}+p\right)} \\ \Lambda & \phi \mu c_{\mathrm{g}}+\frac{p_{\mathrm{sc}} T \mu z}{T_{\mathrm{sc}} q_{\mathrm{D}} p_{i}^{2}} \\ \omega & \frac{\phi \mu c_{\mathrm{g}}}{\Lambda} \\ \tau & \frac{R^{2}}{\mathrm{D}} \\ \lambda & \frac{k \tau}{\Lambda L_{f}^{2}} \\ \mathrm{f}(s) & \omega s+\frac{(1-\omega)}{\lambda} \sigma(\sqrt{\lambda s} \cot h \sqrt{\lambda s}-1) \\ \gamma & \sqrt{f(s)}\end{array}$

\section{Dimensionless variables}

$\begin{array}{ll}\Psi_{D} & \frac{p_{i} \pi k h T_{s c}}{\mu_{i} z_{i} p_{s c} q_{s c} T}\left(\Psi_{i}-\Psi\right) \\ \mathrm{t}_{D} & \frac{k t}{\Lambda L_{f}^{2}} \\ \mathrm{~L}_{D} & \frac{L}{L_{f}}\end{array}$

\section{Introduction}

Coal seams are classified as unconventional gas reservoirs, together with tight gas sands and gas shales. Coalbed methane reservoirs have a dual porosity nature with macrospores made by a natural fracture network, and microspores which exist within the coal matrix. Natural fracture systems consist of two fissure systems, namely, butt and face cleats. Butt cleats are less continuous and often terminate against the face cleat with a strike angle of 75-85 degrees (Anbarci and Ertekin, 1990).

Gas flow in a coal seam involves two mechanisms: diffusion and seepage. When the coal seam pressure drops to a certain degree, the methane in the matrix translates from the adsorbed state into the free state, diffuses into the natural fracture network, and then moves through the hydraulic fracture into the wellbore. The relationship between adsorption volume and pressure can be described by the
Langmuir isothermal adsorption equation. The diffusion process follows the Fick diffusion law. Flow from the natural fracture network to the hydraulic fracture is a Darcy flow. The seepage characteristics of coalbed methane make the mathematical model of seepage more complex.

Since hydraulic fracturing technology is widely and effectively utilized to enhance production efficiency in coalbed methane reservoirs, determining how to evaluate fracturing results is becoming increasingly important. During the past decades, many scholars have contributed to analyzing production and pressure performance, as it is an essential part of reservoir evaluation. Pressure performance analysis also constitutes the basis for production analysis (Mcguire and Sikora, 1960; Raghavan et al., 1972; Albinali et al., 2016; de Swaan, 2016).

Pressure analysis of coalbed methane was commonly performed based on the dual-porosity model that was introduced by Warren and Root (1962), de Swaan O (1976) and Kazemi et al. (1992). Anbarci and Ertekin (1990) proposed the Langmuir isothermal adsorption equation and Fick diffusion law to represent the adsorption and diffusion processes in seepage flow, and to analyze pressure and production performance. Gringarten et al. (1975) developed a transient pressure performance analysis and type curve analysis for fractured wells. Their work identified three basic solutions for fractured wells: infinite-conductivity solution, uniform flux solution for vertical wells, and uniform flux solution for horizontal wells.

However, as the number of segments and fracture halflength increase, the conductivity of the fracture cannot be ignored. Cinco-Ley et al. (1978), Cinco-Ley and Meng (1988) developed a semi-analytical solution for finiteconductivity vertical fractured wells. Aminian and Ameri (2009) proposed a numerical coalbed methane model to predict production behavior of coalbed methane reservoirs. Clarkson et al. (2009) made a production decline curve analysis for fractured wells and horizontal wells in coalbed methane reservoirs. King et al. (1986) used a numerical method to simulate transient pressure performance with finite- and infinite-conductivity vertical fractures in coal seams. Nie et al. (2012) developed an analytical solution to describe its flow characteristics in a horizontal well with multistage fracturing in coalbed methane reservoirs.

Most of the above literature modeled the transport characteristics of vertical and horizontal wells with radial boundaries. However, according to field test results, some fractured wells exhibit the characteristics of the rectangular boundary. Thus, the objective of this paper was to establish a complete semi-analytical model for vertical wells with finite-conductivity fractures in a rectangular-boundary coal seam.

In this paper, we established and solved a new mathematical model with rectangular boundary, adsorption, desorption, diffusion, and finite conductivity fracture. Moreover, wellbore storage effects and skin factor can be added to the solution conveniently. Based on the proposed mathematical model, we optimized the calculation process to improve computational efficiency. Secondly, the model was validated with the literature results and a numerical simulator. Thirdly, the flow characteristics were analyzed. Using parameter sensitivity analysis, the type curves of a 


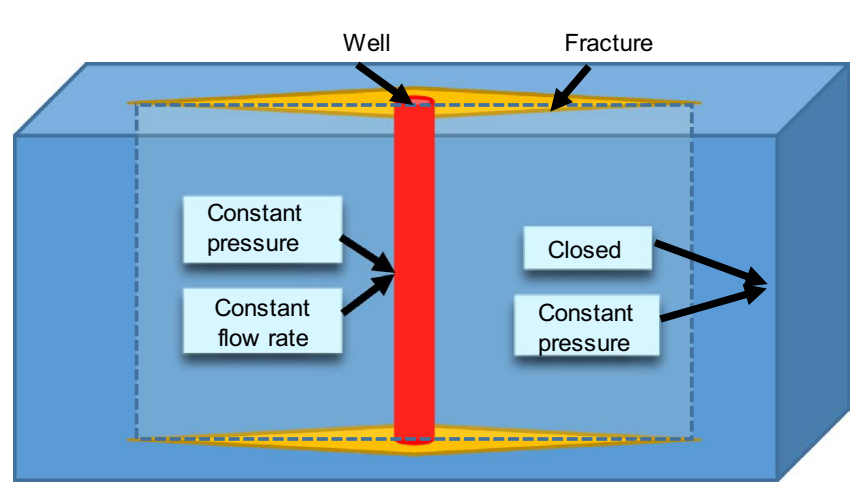

Fig. 1. Reservoir model with rectangular boundary.

finite conductivity fracture vertical well in a rectangular coalbed methane reservoir were established in the paper. Parameters were analyzed, including inter-porosity coefficient, storage coefficient, desorption coefficient, fracture conductivity factor, wellbore storage coefficient, and skin factor. Finally, we applied the newly established model and calculation process to analyze the production and pressure performance of a well from the Qinshui Basin.

\section{Methodology}

Some important assumptions were made for mathematic model development:

1. the well is in the center of a rectangular reservoir that is bounded by upper and lower impermeable layers;

2. the inner boundary has either constant flow rate or constant pressure (Fig. 1);

3 . adsorption in the coal matrix obeys the Langmuir isotherm adsorption equation, diffusion from the matrix to the natural fracture network obeys the Fick law, flow from the natural fracture network to the hydraulic fracture and flow to the wellbore obey the Darcy law (Fig. 2);

4. the well is intercepted by a fully penetrating fracture, and the flow rate in the fracture is different along the fracture (Fig. 3);

5. the reservoir is isotropic and homogeneous with a constant height, porosity, and permeability;

6 . the initial reservoir is uniform;

7. gas flow takes place only through fractures;

8. there is no pressure loss along the wellbore;

9. pressure gradients are so small that the gravity effect is negligible; and

10. wellbore storage effect and skin effect are nonnegligible.

\subsection{Model establishment and solution}

Anbarci and Ertekin (1990) obtained a series of solutions for pressure transient behavior in coal seams for various outer- and inner-boundary conditions. However, their solutions only work for vertical, unfractured, and radial flow. In this article, to establish a sound continuity equation for coalbed methane reservoirs, we considered the flow mechanism of free gas and adsorbed gas separated from the matrix and fractures. According to mirror image theory, the seepage condition of the one well with one fault in the reservoir is completely identical to that of two wells in the reservoir. One well is the same as the original well, and the other well is a reflected duplication of the original well that appears almost identical, but is reversed in the direction perpendicular to the fault surface. Using the Laplace transformation and the mirror image theory, we can obtain the solution of the continuity equation from Ozkan and Raghavan (1988, 1991) source solution.

\subsubsection{Modeling flow in the reservoir}

In the material balance equation of coalbed methane reservoirs, besides free gas flow, adsorbed gas separates from the matrix and participates in the flow, which is different from conventional gas. The continuity equation is defined as equation (1):

$$
\frac{1}{r} \frac{\partial}{\partial r}\left(r \frac{p}{\mu z} \frac{\partial p}{\partial r}\right)=\frac{\varphi c_{\mathrm{g}} p}{k z} \frac{\partial p}{\partial t}+\frac{p_{\mathrm{sc}} T}{k T_{\mathrm{sc}}} \frac{\partial V}{\partial t} .
$$

Gas seepage from matrix to natural fracture network was usually expressed by the Fick diffusion law:

$$
\frac{1}{r^{2}} \frac{\partial}{\partial r}\left(r^{2} D \frac{\partial C}{\partial r}\right)=\frac{\partial C}{\partial t} .
$$

The Langmuir isothermal adsorption equation describes the desorption from adsorbed gas to free gas:

$$
C=\frac{V_{\mathrm{L}} p}{p_{\mathrm{L}}+p} .
$$

Matrix geometry is assumed to be composed of spherical blocks with radius $R$. The unsteady-state sorption equation for the coal matrix can be written as:

$$
\frac{\partial V}{\partial t}=\left.\frac{3 D}{R} \frac{\partial C}{\partial r_{i}}\right|_{r_{i}=R}
$$

Inner boundary condition in the dimensionless form for the microspore equation is:

$$
\frac{\partial C_{D}}{\partial r_{D i}}\left(r_{D i}=0, t_{D}\right)=0 .
$$

Outer boundary condition in the dimensionless form for the microspore equation is:

$$
C_{D}\left(r_{D i}=1, t_{D}\right)=\left.C_{D}\right|_{p_{D a}\left(r_{D a}, t_{D}\right)} .
$$

The solution for equation (2):

$$
\frac{\mathrm{d} \tilde{C}_{D}}{\mathrm{~d} r_{D i}}\left(r_{D i}=1\right)=\tilde{C}_{D a}(\sqrt{\lambda s} \cot h \sqrt{\lambda s}-1) .
$$

Combining with equation (4), one can linearize equation (1). 


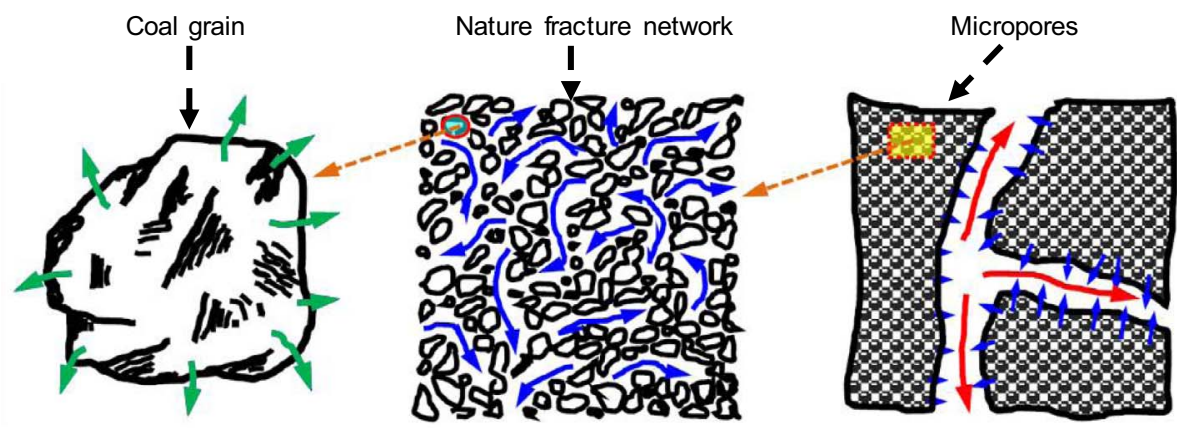

Desorption from a coal frame Flow in the nature fracture network Flow in the fracture network

Fig. 2. Desorption, diffusion, and Darcy flow in the matrix and fracture.

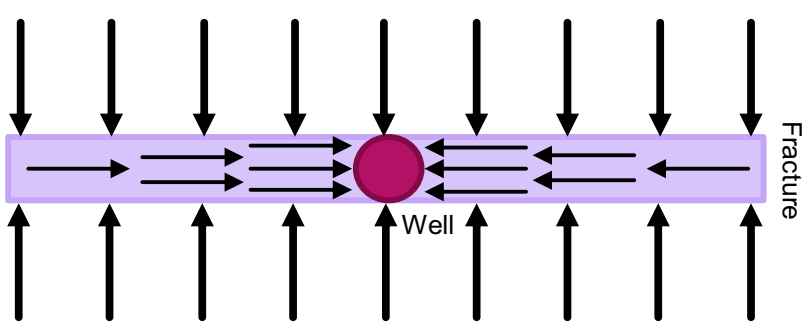

Fig. 3. Flow in the finite-conductivity fracture.

It is required for $\tilde{p_{D}}$ to vanish as $r_{D} \rightarrow \infty$ for $t_{D} \geq 0$ and at $t_{D}=0$ for $r_{D}>\epsilon(\epsilon \rightarrow 0+)$. These conditions are expressed by:

$$
\begin{gathered}
\tilde{p_{D}}\left(t_{D} \geq 0, r_{D} \rightarrow \infty\right)=0 \\
\tilde{p}_{D}\left(t_{D}=0, r_{D}>\epsilon(\epsilon \rightarrow 0+)\right)=0 .
\end{gathered}
$$

Normalized pseudo-pressure defined by Meunier et al. (1984) is:

$$
\psi=\frac{\mu_{i} z_{i}}{p_{i}} \int_{0}^{p} \frac{p}{\mu z} \mathrm{~d} p
$$

The final continuity equation in the Laplace domain is:

$$
\frac{1}{r_{D}} \frac{\partial}{\partial r_{D}}\left(r_{D} \frac{\partial \tilde{\psi}_{D}}{\partial r_{D}}\right)=f(s) \tilde{\psi}_{D}
$$

The closed boundary or constant pressure conditions in a rectangular coordinate system are:

$$
\begin{gathered}
\left.\left(\frac{\partial \tilde{\psi}_{D}}{\partial x_{D}}+\frac{\partial \tilde{\psi}_{D}}{\partial y_{D}}\right)\right|_{\left(x_{D}=x_{e D}, y_{D}=y_{e D}\right)}=0 \\
\left.\tilde{\psi}_{D}\right|_{\left(x_{D}=x_{e D}\right)}=0 \\
\left.\tilde{\psi}_{D}\right|_{\left(y_{D}=y_{e D}\right)}=0 .
\end{gathered}
$$

Translating a radial coordinate system to a rectangular coordinate system as:

$$
r_{D}=\sqrt{x_{D}^{2}+y_{D}^{2}}
$$

Ozkan and Raghavan (1991) presented solutions for a rectangular closed and constant-pressure outer boundary in the conventional reservoir as follows.

Rectangular closed outer boundary:

$$
\begin{aligned}
& \tilde{p}_{D}\left(x_{D}, y_{D}\right)=\frac{\pi}{2 x_{e D}} \tilde{q}_{f D}\left[\frac{\cos h\left(\sqrt{s} y_{D 1}\right)+\cos h\left(\sqrt{s} y_{D 2}\right)}{\sqrt{s} \sin h\left(\sqrt{s} y_{e D}\right)}\right. \\
& +2 \sum_{k=1}^{\infty} \frac{1}{x_{e D}} \cos \left(k \pi \frac{x_{w D}}{x_{e D}}\right) \cos \left(k \pi \frac{x_{D}}{x_{e D}}\right) \frac{\cos h\left(\varepsilon_{k} y_{D 1}\right)+\cos h\left(\varepsilon_{k} y_{D 2}\right)}{\varepsilon_{k} \sin h\left(\varepsilon_{k} y_{e D}\right)} \\
& +2 \sum_{n=1}^{\infty} \cos \left(n \pi \frac{z_{D}}{h_{D}}\right) \cos \left(n \pi \frac{z_{w D}}{h_{D}}\right)\left(\frac{\cosh \left(\varepsilon_{n} y_{D 1}\right)+\cos h\left(\varepsilon_{n} y_{D 2}\right)}{\varepsilon_{n} \sin h\left(\varepsilon_{n} y_{e D}\right)}\right. \\
& \left.\left.+2 \sum_{k=1}^{\infty} \cos \left(k \pi \frac{x_{D}}{x_{e D}}\right) \cos \left(k \pi \frac{x_{w D}}{x_{e D}}\right) \frac{\cosh \left(\varepsilon_{k, n} y_{D 1}\right)+\cos h\left(\varepsilon_{k, n} y_{D 2}\right)}{\varepsilon_{k, n} \sin h\left(\varepsilon_{k, n} y_{e D}\right)}\right)\right] .
\end{aligned}
$$

Rectangular constant-pressure outer boundary:

$$
\begin{aligned}
& \tilde{p}_{D}\left(x_{D}, y_{D}\right)=\frac{\pi}{x_{e D}} \tilde{q}_{f D}\left[\sum_{k=1}^{\infty} \sin \left(k \pi \frac{x_{D}}{x_{e D}}\right) \sin \left(k \pi \frac{x_{w D}}{x_{e D}}\right)\right. \\
& \frac{\cos h\left(\varepsilon_{k} y_{D 1}\right)-\cos h\left(\varepsilon_{k} y_{D 2}\right)}{\varepsilon_{k} \sin h\left(\varepsilon_{k} y_{e D}\right)} \\
& +2 \sum_{n=1}^{\infty} \cos \left(n \pi \frac{z_{D}}{h_{D}}\right) \cos \left(n \pi \frac{z_{w D}}{h_{D}}\right) \sum_{k=1}^{\infty} \sin \left(k \pi \frac{x_{D}}{x_{e D}}\right) \\
& \left.\sin \left(k \pi \frac{x_{w D}}{x_{e D}}\right) \frac{\cos h\left(\varepsilon_{k, n} y_{D 1}\right)-\cos h\left(\varepsilon_{k, n} y_{D 2}\right)}{\varepsilon_{k, n} \sin h\left(\varepsilon_{k, n} y_{e D}\right)}\right] .
\end{aligned}
$$

Different from conventional gas reservoirs, coalbed seams have strong adsorption and desorption with the seepage. So, we must reflect this phenomenon in the control equation (Eq. (1)). The new parameter $\gamma$ (the specific expression shown in Intermediate Variables) will reveal this 
specific phenomenon in the coalbed seam. Combining with it, we obtain the solutions in the coal seam as follows.

Rectangular closed outer boundary:

$\tilde{p}_{D}\left(x_{D}, y_{D}\right)=\frac{\pi}{2 x_{e D}} \tilde{q}_{f D}\left(\frac{\cosh \left(\gamma y_{D 1}\right)+\cosh \left(\gamma y_{D 2}\right)}{\gamma \sin h\left(\gamma y_{e D}\right)}\right.$

$\left.+2 \sum_{k=1}^{\infty} \frac{1}{x_{e D}} \cos \left(k \pi \frac{x_{w D}}{x_{e D}}\right) \cos \left(k \pi \frac{x_{D}}{x_{e D}}\right) \frac{\cos h\left(\varepsilon_{k} y_{D 1}\right)+\cos h\left(\varepsilon_{k} y_{D 2}\right)}{\varepsilon_{k} \sin h\left(\varepsilon_{k} y_{e D}\right)}\right)$.

Rectangular constant-pressure outer boundary:

$$
\begin{aligned}
\tilde{p}_{D}\left(x_{D}, y_{D}\right)= & \frac{\pi}{x_{e D}} \tilde{q}_{f D} \sum_{k=1}^{\infty} \sin \left(k \pi \frac{x_{D}}{x_{e D}}\right) \sin \left(k \pi \frac{x_{w D}^{\prime}}{x_{e D}}\right) \\
& \frac{\cosh \left(\varepsilon_{k} y_{D 1}\right)-\cos h\left(\varepsilon_{k} y_{D 2}\right)}{\varepsilon_{k} \sin h\left(\varepsilon_{k} y_{e D}\right)}
\end{aligned}
$$

where

$$
\begin{aligned}
\epsilon_{n} & =\left(\gamma^{2}+n^{2} \pi^{2} / h_{D}^{2}\right)^{1 / 2}, y_{D 1}=y_{e D}-\left|y_{D}-y_{w D}\right|, y_{D 2} \\
& =y_{e D}-\left|y_{D}+y_{w D}\right|, y_{D}=y_{w D}=y_{e D} / 2 .
\end{aligned}
$$

\subsubsection{Modeling flow in the fracture}

Taking equation (18) to integral along the fracture direction yields:

$$
\begin{aligned}
\tilde{\psi}_{D}\left(x_{D}, y_{D}\right)= & \frac{\pi}{2 x_{e D}} \int_{x_{w D}-1}^{x_{w D}+1} \tilde{q}_{f D} \frac{\cos h\left(\gamma y_{D 1}\right)+\cos h\left(\gamma y_{D 2}\right)}{\gamma \sin h\left(\gamma y_{e D}\right)} \mathrm{d} \alpha \\
& +\frac{\pi}{x_{e D}} \int_{x_{w D}-1}^{x_{w D}+1} \tilde{q}_{f D} \sum_{k=1}^{\infty} \frac{1}{x_{e D}} \\
& \times \cos \left(k \pi \frac{x_{w D}}{x_{e D}}\right) \cos \left(k \pi \frac{x_{D}}{x_{e D}}\right) \\
& \frac{\cosh \left(\varepsilon_{k} y_{D 1}\right)-\cosh \left(\varepsilon_{k} y_{D 2}\right)}{\varepsilon_{k} \sin h\left(\varepsilon_{k} y_{e D}\right)} \mathrm{d} \alpha .
\end{aligned}
$$

In this equation, $\tilde{q}_{f D}$ is a function of the location in the fracture. To integral the equation along the fracture, we use:

$$
\begin{aligned}
\alpha & =x_{w D}-x, \mathrm{~d} \alpha=-\mathrm{d} x, x=x_{w D}-\alpha, \int_{x_{w D}-1}^{x_{w D}+1} \tilde{q}_{f D} \mathrm{~d} \alpha \\
& =\int_{-1}^{1} \tilde{q}_{f D} x \mathrm{~d} x .
\end{aligned}
$$

Using this definition, equation (20) can be translated as:

$$
\begin{aligned}
\tilde{\psi}_{D}\left(x_{D}, y_{D}\right) & =\frac{2 \pi}{x_{e D}} \int_{0}^{1} \tilde{q}_{f D} \sum_{k=1}^{\infty} \\
& \cos \left(k \pi \frac{x_{D}}{x_{e D}}\right) \cos \left(\frac{k \pi \alpha}{x_{e D}}\right) \cos \left(\frac{k \pi}{2}\right) \\
& \frac{\cos h\left(\varepsilon_{k} y_{D 1}\right)+\cos h\left(\varepsilon_{k} y_{D 2}\right)}{\varepsilon_{k} \sin h\left(\varepsilon_{k} y_{e D}\right)} \mathrm{d} \alpha .
\end{aligned}
$$

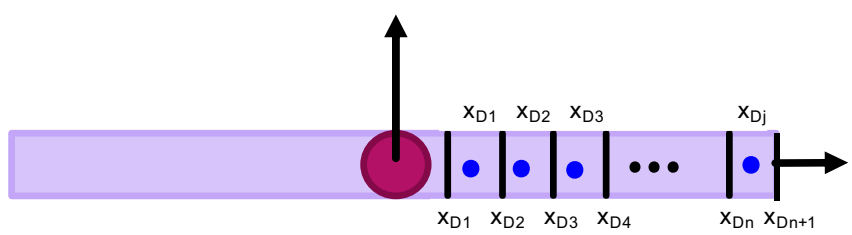

Fig. 4. Discretizing the equation along the fracture.

Assuming that the fracture can be divided into $n$ segments. Discretizing equation (21) with Figure 4 yields:

$$
\begin{aligned}
\tilde{\psi}_{D}\left(x_{D}, y_{D}\right) & =4 \sum_{i=1}^{n} \tilde{q}_{f D} \sum_{k=1}^{\infty} \frac{1}{k} \cos \left(k \pi \frac{x_{D}}{x_{e D}}\right) \cos \left(\frac{k \pi}{2}\right) \\
& \cos \left(k \pi \frac{x_{D i+1}+x_{D i}}{2 x_{e D}}\right) \sin \frac{k \pi \Delta x}{2 x_{e D}} \\
& \times \frac{\cos h\left(\varepsilon_{k} y_{D 1}\right)+\cos h\left(\varepsilon_{k} y_{D 2}\right)}{\varepsilon_{k} \sin h\left(\varepsilon_{k} y_{e D}\right)} .
\end{aligned}
$$

In the Laplace domain, the sum of all of the flow rates in each part is $1 / \mathrm{s}$. So, it can be expressed as:

$$
\Delta x \sum_{i=1}^{n} \tilde{q}_{f D i}(s)=\frac{1}{s}
$$

In the process of discretization, we divided the fracture into $n$ parts. We assumed that the flow rate of each part is different, but the flow rate is constant in the same part. For each part, there is a pressure equation. Combined with the flow rate normalization equation, we can obtain an equation set with $n+1$ equations and $n+1$ unknown numbers as $\tilde{\psi}_{D}, \tilde{q}_{D h f 1}, \tilde{q}_{D h f 2}, \tilde{q}_{D h f 3}, \ldots, \tilde{q}_{D h f n}$. Solving this equation set, the solution of $\psi_{D}$ can be determined.

Duhamel's theory can be utilized to incorporate the wellbore storage coefficient and skin factor into well response. So, the final solution with wellbore storage and skin in the Laplace domain can be written as:

$$
\tilde{\psi}_{s w D}=\frac{1}{s^{2} C_{D}+s\left[s \tilde{\psi}_{w D}(s)+S_{k}\right]} .
$$

\subsubsection{Calculation process optimization}

Three factors lead to the complex calculation of finite-conductivity fractured wells in rectangular coal reservoirs: (1) solution of the rectangular reservoir from the mirror image theory, which produces mirror wells; (2) diffuse gas flowed in the coal seam will increase complexity; and (3) finiteconductivity fracture requires solving an equation set. All of these reasons eventually result in slower computing for the normal solution that is not suitable for software development. The objective was to obtain a quick calculation method to determine the pressure or production for finiteconductivity fractured wells in a rectangular coal seam.

We introduced the fracture conductivity function to translate the infinite-conductivity fracture solution to the 
finite-conductivity fracture. The specific equation translates as Wang et al., (2014):

$$
s \tilde{p}_{w D}=s \tilde{p}_{w D \text { inf }}+s \tilde{f}\left(C_{f D}\right) .
$$

From Riley et al. (1991), $\tilde{f}\left(C_{f D}\right)$ expresses the fracture conductivity as:

$$
\begin{aligned}
s \tilde{f}\left(C_{f D}\right)= & 2 \pi \sum_{n=1}^{\infty} \frac{1}{n^{2} \pi^{2} C_{f D}+2 \sqrt{n^{2} \pi^{2}+s}} \\
& +\frac{0.4063 \pi}{\pi\left(C_{f D}+0.8997\right)+1.6252 s} .
\end{aligned}
$$

\subsubsection{Model verification}

To verify our model and solutions, we compared it with Anbarci and Ertekin's work (1992) and a reservoir simulator- the UNConventional Oil and Gas simulator (UNCONG) (Li et al., 2015). Anbarci and Ertekin (1992) set the model with an infinite reservoir boundary and infinite conductivity fracture. Corresponding to their model, we set a large enough boundary and fracture conductivity factor. UNCONG incorporates comprehensive flowing mechanisms for unconventional reservoirs (Li et al., 2015). The same as Anbarci et al.'s work, basic data used to perform the calculation are listed in Table 1.

Compared the normal solution with Anbarci's analytical solution, the solution presented in this paper fits better with the numerical solution (Fig. 5). It can thus be concluded that the new method to solve the fracture model is more accurate.

From Figure 6, we can see that using the fracture conductivity function method can improve computation speed greatly. In fact, it is 488 times faster with a closed boundary, and 599 times faster with a constant-pressure boundary. For the closed boundary, the relative error was $1.25 \%$, and for the constant pressure boundary, the relative error was $3.23 \%$. So, this function can be used for rectangular boundaries to dramatically increase calculation speed.

\section{Results and discussion}

\subsection{Flow characteristics analysis of type curves}

Because of the two different sets of borders, pressure reaches the boundary twice. Under this condition, the flow state is different from square or circle boundaries. It can be divided into seven stages (Fig. 7):

1. Wellbore storage stage. This stage mainly reflects the effect of wellbore storage at an early time. The linear slope of both dimensionless pseudo-pressure and its derivative is 1 .

2. Transition stage of wellbore storage. In this stage, the wellbore storage influence decreases, and the gas in the reservoir flows into the wellbore gradually. The pressure is influenced by the reservoir and the wellbore storage, and the curve of the dimensionless pseudo-pressure derivative decreases.
Table 1. Basic data to verify results.

\begin{tabular}{lclc}
\hline$\tau(\mathrm{hr})$ & 328990 & $r_{\mathrm{w}}(\mathrm{ft})$ & 0.5 \\
$\mu(\mathrm{cp})$ & 0.01082 & $c\left(\mathrm{psia}^{-1}\right)$ & 0.002234 \\
$T(\mathrm{R})$ & 530 & $z$ & 0.9404 \\
$q_{\mathrm{sc}}(\mathrm{MMscf} / \mathrm{d})$ & 0.2 & $V_{\mathrm{L}}\left(\mathrm{scf} / \mathrm{ft}^{3}\right)$ & 18.632 \\
$\mathrm{p}_{\mathrm{ic}}(\mathrm{psia})$ & 447.7 & $k(\mathrm{md})$ & 26 \\
$\varphi$ & 0.01 & $h(\mathrm{ft})$ & 6 \\
$x_{\mathrm{f}}(\mathrm{ft})$ & 100 & $P_{\mathrm{L}}(\mathrm{psi})$ & 167.58 \\
\hline
\end{tabular}

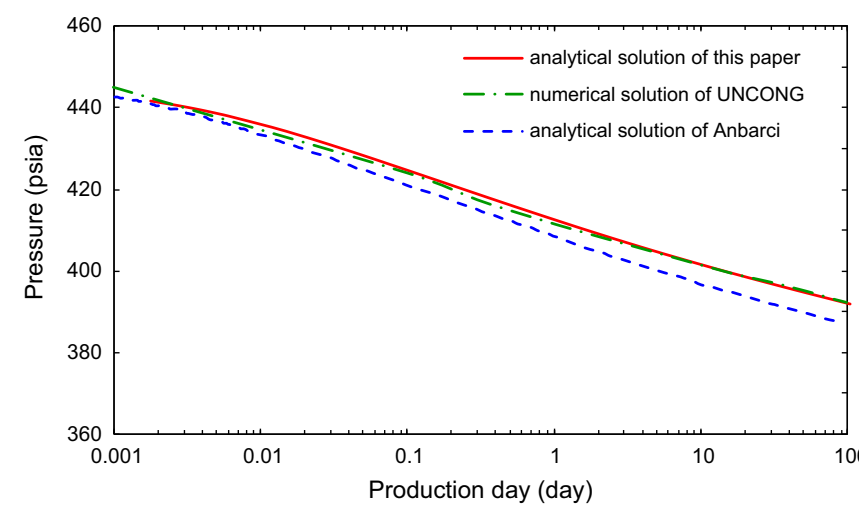

Fig. 5. Comparison between Anbarci analytical and numerical solution.

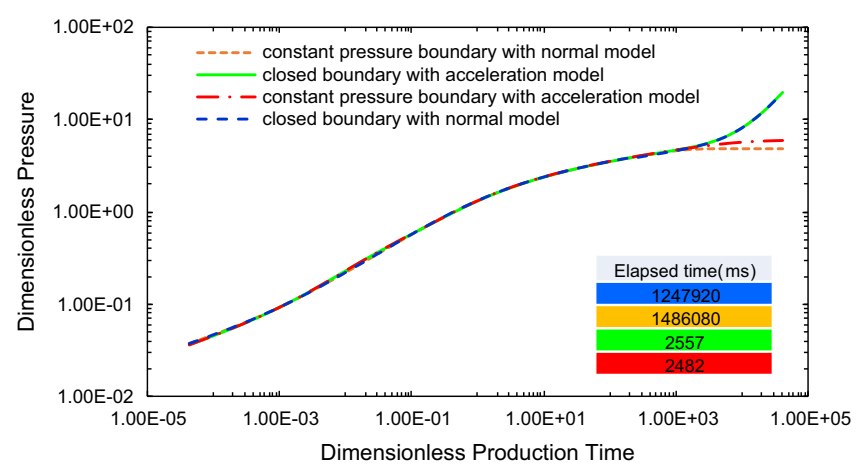

Fig. 6. Comparison between normal and conductivity function calculations.

3. Linear flow stage from natural fracture to hydraulic fracture. This stage can be divided into two substages: bilinear flow and linear flow. The slope of the dimensionless pseudo-pressure derivative curve changes from $1 / 4$ to $1 / 2$. Fracture conductivity determines the length of bilinear flow. Specifically, the larger the conductivity, the longer the duration time. The dimensionless pseudo-pressure decreases slightly in the reservoir in this stage; the diffusion phenomenon is not obvious.

4. Desorption and diffusion stage. With the pressure decreasing, desorption becomes increasingly obvious. The adsorbed gas desorbed from the matrix becomes 


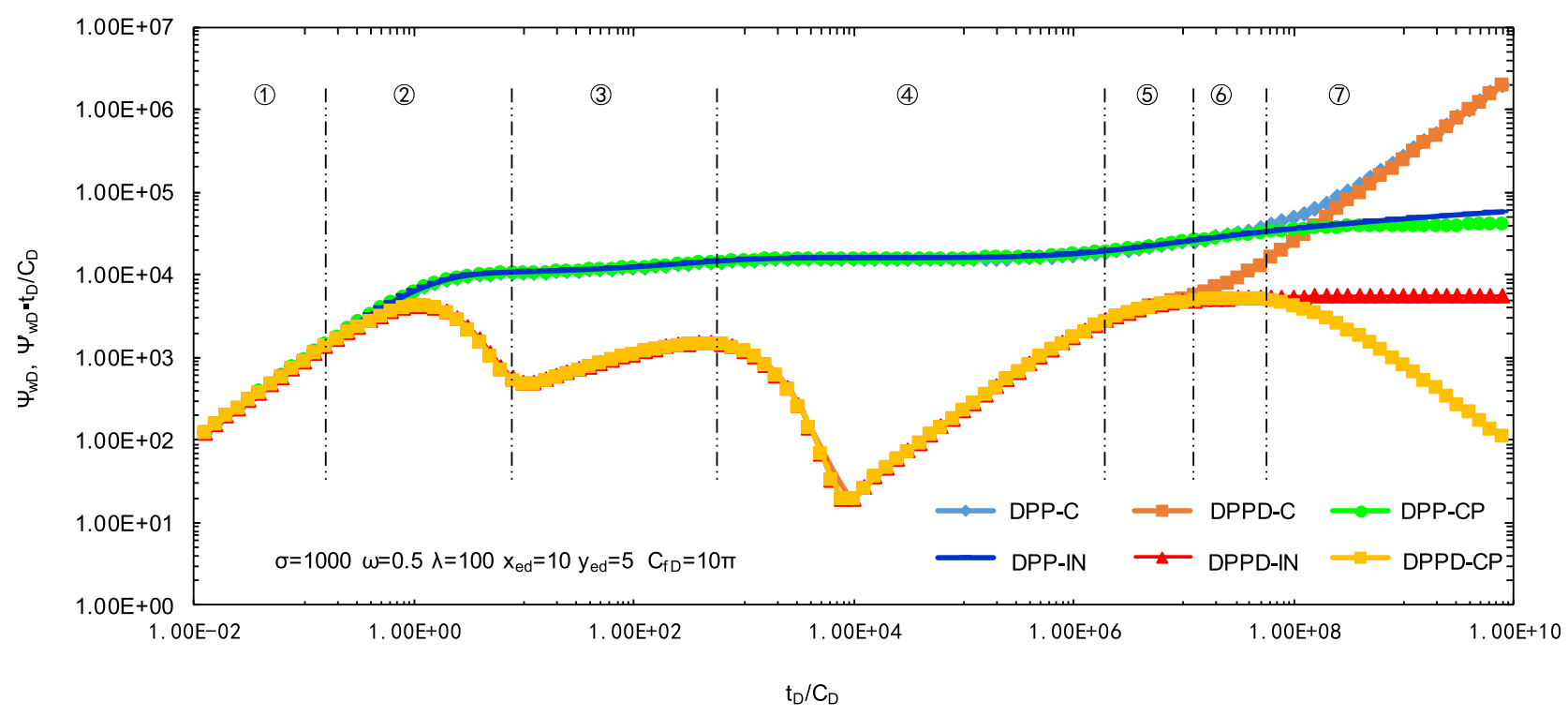

Fig. 7. Flow stage divided for boxed coal reservoirs.

free gas, leading to concentration increase around the matrix and diffusion to the natural fracture. This stage reflects the dimensionless pseudo-pressure appearing in a "V" shape; its depth and appearance time are influenced by the wellbore storage coefficient, interporosity coefficient, storage coefficient, and desorption coefficient. This stage constitutes the important flow stage that reflects the characteristics of coalbed methane.

5. Pseudo-radial flow stage. In this stage, the gas diffused from the matrix has dynamic equilibrium with the gas flowing into the wellbore. The speed of pressure decline becomes slow.

6. First boundary control flow stage. At this time, the pressure wave reaches the first boundary. With the constant-pressure boundary, the pressure reaches stabilization quickly. However, the closed boundary has a transition, and so the dimensionless pseudo-pressure and derivative reflect gradually. This is because the constant-pressure boundary has a sufficient energy supply to enable the pressure to reach a balance in a short time.

7. Second boundary control flow stage. At this time, the constant-pressure boundary has already reached balance in the first boundary control flow stage, but the closed boundary must reach the pseudo-steady state again.

In these seven stages, the linear flow stage mainly reflects the characteristics of fractured vertical wells and the conductivity of a hydraulic fracture. The desorption and diffusion stage mainly reflect the characteristics of an unconventional gas reservoir that has the phenomenon of desorption and diffusion, such as coalbed methane and shale gas. The two boundary control flows mainly reflect the characteristics of a rectangular reservoir. In this picture, $\mathrm{C}$ represents the closed boundary type, CP represents the
Table 2. Basic factors for sensitivity analysis.

\begin{tabular}{llllll}
\hline$\lambda$ & 50 & $\sigma$ & 500 & $\omega$ & 0.5 \\
$S_{\mathrm{k}}$ & 0.1 & $\mathrm{CD}$ & 0.0001 & $\mathrm{C}_{\mathrm{fD}}$ & $10 \pi$ \\
\hline
\end{tabular}

constant pressure boundary type, IN represents the infinite boundary type.

\subsection{Sensitivity analysis}

Based on the definition above, traditional factors, such as permeability, porosity and compressibility, were regrouped as new factors, such as interporosity coefficient, storage coefficient, and desorption coefficient (shown in Intermediate Variables). Combined with the fracture conductivity factor, wellbore storage coefficient and skin factor, we performed a sensitivity analysis on those six factors (Tab. 2).

Interporosity coefficient $\lambda$ is the direct ratio of permeability and time constant, and the inverse ratio of porosity, viscosity, fracture half-length, and gas compressibility. Figure 8a gives the effect of $\lambda$ on coal seams with closed boundary conditions. It can be seen from the curves that a greater $\lambda$ will lead to a smaller time of concave-down characteristic appearance. The $\lambda$ slightly influences the degree of desorption and diffusion.

Storage coefficient $\omega$ is the direct ratio of porosity; it reflects the storage ability of the fracture system. It has an inverse effect compared with the conventional dual porosity medium model reflecting the matrix storage capacity. Figure $8 \mathrm{~b}$ gives the effect of $\omega$ on coal seams with closed boundary conditions. The larger the $\omega$, the greater the porosity of the fracture and the gas flow rate from the fracture, the smaller the gas flow rate from the matrix diffusion. Pressure maintenance due to desorption is more obviously marked as $\omega$ becomes smaller. However, the effect is inverse 


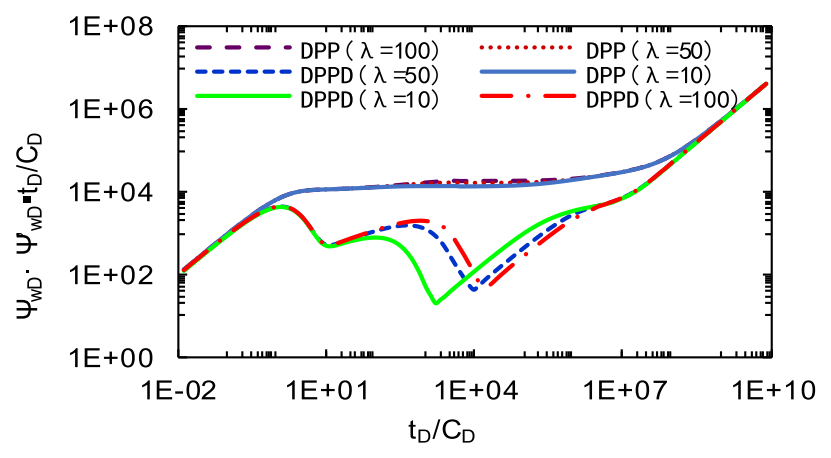

(a) Influence of interporosity coefficient

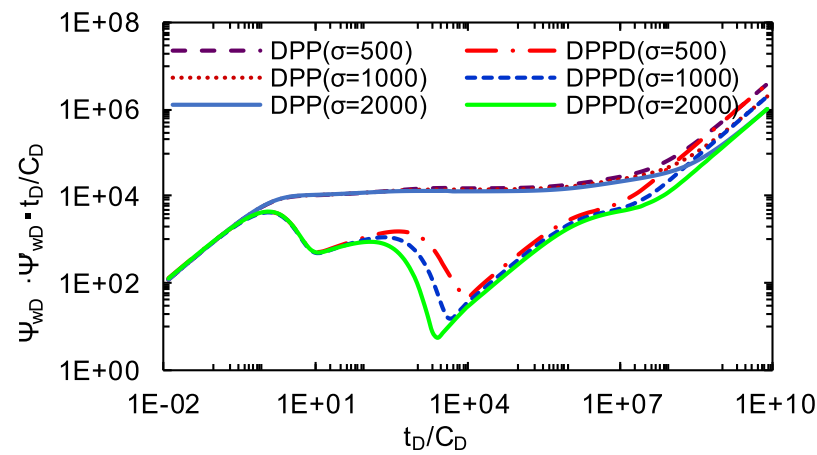

(c) Influence of desorption coefficient

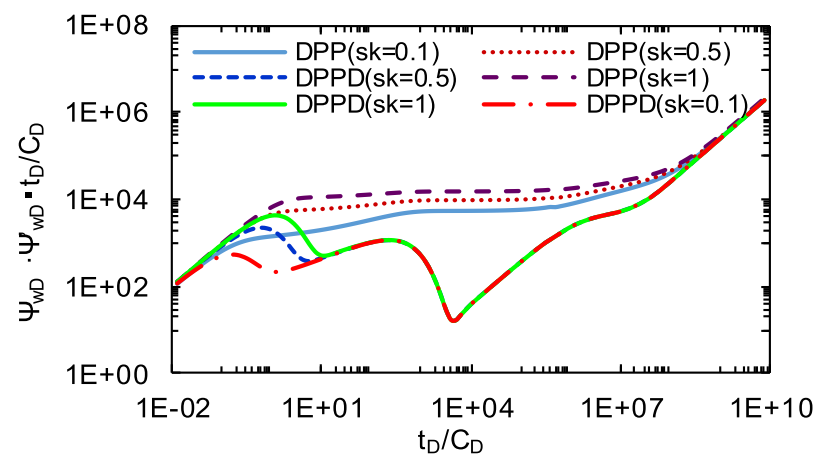

(e) Influence of skin factor

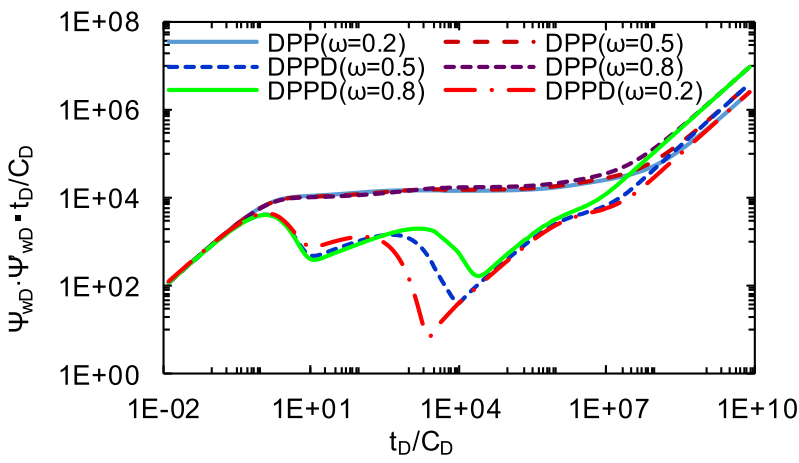

(b) Influence of storage coefficient

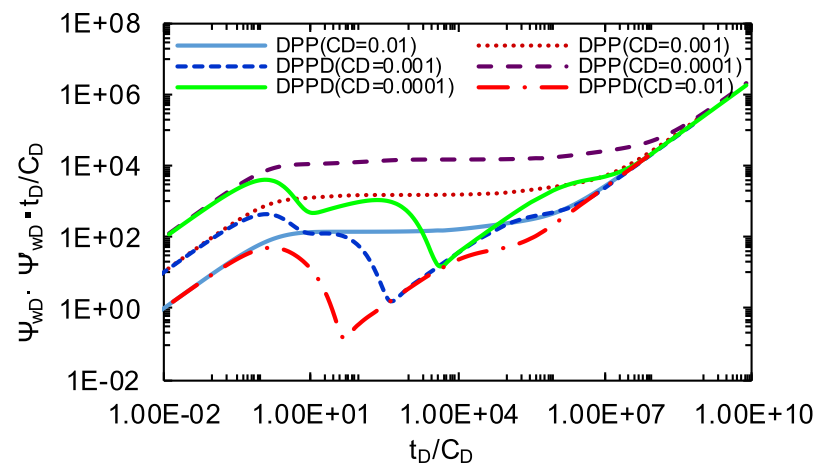

(d) Influence of well storage

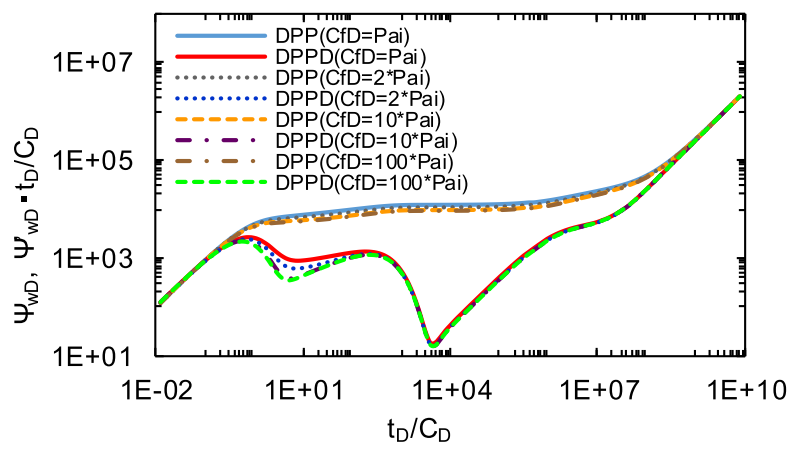

(f) Influence of fracture conductivity

Fig. 8. Influence of parameters on type curve. (a) Influence of interporosity coefficient; (b) Influence of storage coefficient; (c) Influence of desorption coefficient; (d) Influence of well storage; (e) Influence of skin factor; (f) Influence of fracture conductivity.

at an early time due to the flow occurring in the natural fractures.

Desorption coefficient $\sigma$ mainly describes the capacity of desorption and diffusion. It constitutes a direct ratio of Langmuir volume, flow rate, and original pressure. Figure $8 \mathrm{c}$ gives the effect of $\sigma$ on coal seams with closed boundary conditions. It can be seen from the curves that a greater $\sigma$ will lead to more gas that the rock can adsorb and produce. In other words, a quicker change of gas concentration leads to an enhanced desorption and diffusion effect, so as to make the concave-down phenomenon become obvious and early.

Well storage coefficient mainly affects the early stage of production. Figure 8d gives the effect of well storage coefficient on coal seams with closed boundary conditions. It can be seen from the curves that a larger coefficient leads to a more obvious well storage effect and longer well storage stage. If the well storage coefficient reaches a certain value, it covers up the linear flow phase, making the flow move directly into the matrix methane diffusion phase.

Skin factor mainly affects pressure performance for wellbore storage and its transition stage, primarily influencing the end time of this stage and dimensionless pseudopressure decrease. Figure 8e gives the effect of skin factor on coal seams with closed boundary conditions. It can be seen from the curves that a larger skin factor will prolong the time of the wellbore storage stage. This is because, at the same flow rate conditions, the higher skin factor will lead to more energy consumed and pressure decreased. 


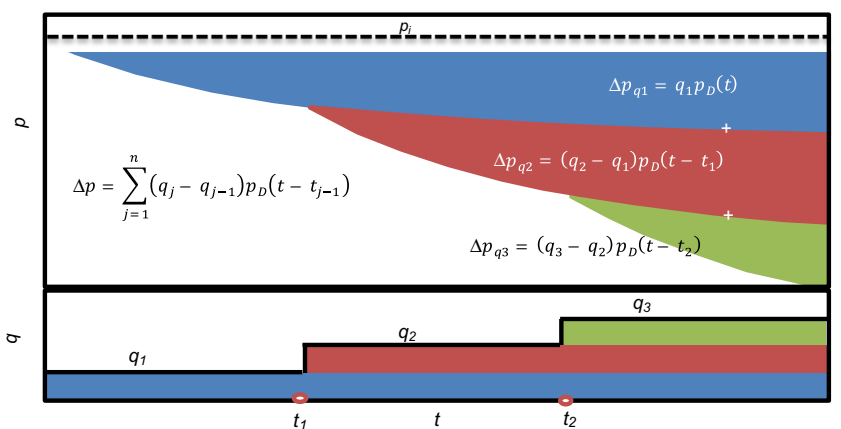

Fig. 9. The principle diagram of Duhamel convolution.

After this stage, the skin factor does not influence pressure performance.

Fracture conductivity factor mainly influences the transition stage of wellbore storage and the linear flow stage; it has no influence on the wellbore storage stage or after the linear flow stage. This is because those stages have low gas flow coming from the natural fracture to the hydraulic fracture. At this time, the finite-conductivity fracture can lead these gas flows to the wellbore quickly, and can be equivalent to the infinite-conductivity fracture. In addition, with the fracture conductivity factor increasing to four times of $\mathrm{Pi}(\mathrm{Pi}=3.14)$, the finite-conductivity fracture gets close to the infinite-conductivity fracture (Fig. 8f). This will guide us to not blindly improve fracture conductivity to improve output; this will not only not influence output, but will increase fracturing cost.

\subsection{Production analysis}

Duhamel convolution is used to construct complex solutions (such as variable rates or pressure) with simple solutions (such as constant rates or pressure). Superposition uses the theory that a rate that changes from $q_{1}$ at time $t$ to a new rate $\mathrm{q}_{2}$ is equivalent to $\mathrm{q}_{1}$ continuing forever, superposed, or added on $\left(\mathrm{q}_{2}-\mathrm{q}_{1}\right)$ starting at time $t$ and continuing forever (Fig. 9).

The steps of the Duhamel convolution are as follows: (1) determine the average pressure through the material balance equation; (2) normalize the production time; (3) calculate the dimensionless pressure or rates through the analytical model and time superposition; and (4) obtain the rate or bottomhole pressure through a lookup table.

\subsection{Field application}

\subsubsection{Basic data}

The proposed model was used to analyze production data from a fractured vertical well in a coal methane reservoir located in the Qinshui Basin in Shanxi Province of China. Daily production data, flowing wellhead pressure measurements (converted to bottomhole pressure for analysis), and some reservoir parameters were collected (Tab. 3). From the seismic data analysis, the well located in the closed rectangle boundary. We used the proposed model in this paper to match the reservoir pressure and the flow rate.
Table 3. Basic data of actual field well.

\begin{tabular}{lclc}
\hline $\mathrm{p}_{\text {ic }}(\mathrm{psia})$ & 340 & $s$ & 0.37 \\
$k(\mathrm{md})$ & 0.05 & $\rho_{\mathrm{b}}\left(\mathrm{g} / \mathrm{cm}^{3}\right)$ & 1.47 \\
$x_{\mathrm{e}}(\mathrm{ft})$ & 1027 & $y_{\mathrm{e}}(\mathrm{ft})$ & 2200 \\
$h(\mathrm{ft})$ & 30 & $C_{\mathrm{t}}\left(\mathrm{psi}^{-1}\right)$ & 0.0031 \\
$R_{\mathrm{w}}(\mathrm{ft})$ & 0.3 & $T(\mathrm{~F})$ & 57.2 \\
$\varphi$ & 0.2 & $V_{\mathrm{L}}(\mathrm{scf} /$ ton $)$ & 321 \\
$L_{\mathrm{f}}(\mathrm{ft})$ & 365 & $P_{\mathrm{L}}(\mathrm{psi})$ & 167.58 \\
\hline
\end{tabular}

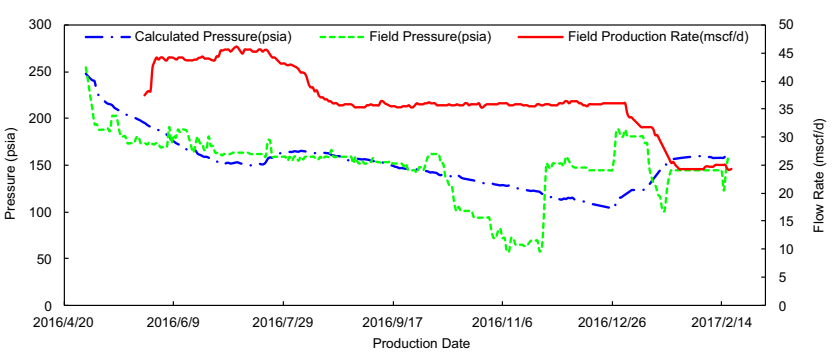

(a) History match of pressure

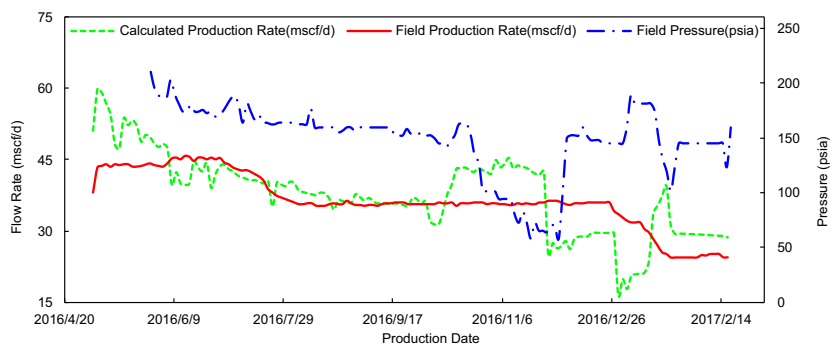

(b) History match of flow rate

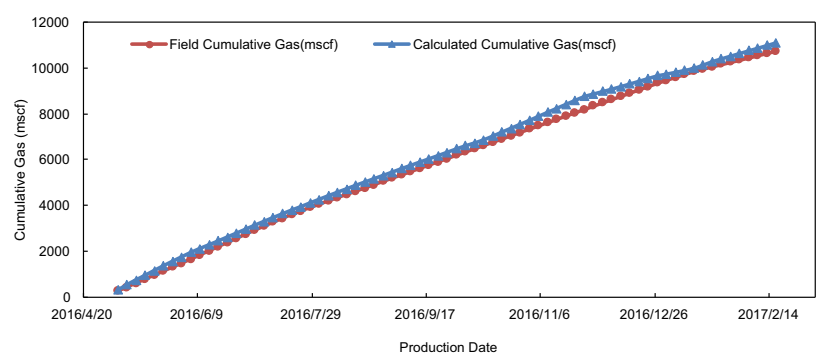

(c) History match of cumulative gas production

Fig. 10. History match result. (a) History match of pressure; (b) History match of flow rate; (c) History match of cumulative gas production.

\subsubsection{History match result}

Using the model in this paper, and combining with the principle of time superposition and the dimensional result from the model, we can obtain the match result. Figure 10a showed the pressure matching. The average error was $18 \%$. Some additional factor may have resulted in the large error at the end of the match.

Figure 10b presented the flow rate matching. The average error was $17 \%$. The same as the pressure match, some additional factor may have resulted in the large error at the end of the match. Figure 10c showed the cumulative 
gas of the calculated value and field value. There was a good match result between the calculated value and field value.

\section{Conclusion}

In this paper, we developed a rectangular-boundary model for a finite-conductivity fractured well in a coal seam. With the mirror image theory and Laplace transformation, the solutions of this model were obtained at a constant production rate or bottomhole pressure. Through the above analysis, the following conclusions can be summarized:

1. By comparing the solution of the simplified model in this paper with the classical analytical model and numerical simulation model, the results show that the proposed method is more accurate and efficient to solve the fractured model and has a good match with the numerical solution.

2. Fracture conductivity function can dramatically improve computation speed without sacrificing accuracy for a rectangular boundary model.

3. Compared with the radial boundary, the new rectangular model has two new flow stages: the first and second boundary control flow stages, which results from the characteristics of rectangular reservoirs. Closed and constant pressure boundaries show the difference stabilization time in pressure performance: pressure reaches stabilization quickly with the constant-pressure boundary, but the closed boundary has a transition after reaching the first boundary, and reaches stabilization after reaching the second boundary.

4. Interporosity coefficient influences the appearance time to desorption and diffusion, and slightly influences the degree. Storage coefficient influences the degree of desorption and diffusion, and slightly influences the appearance time. Desorption coefficient mainly describes the capacity of desorption and diffusion. Well storage coefficient and skin factor mainly affect the early stage of production. Conductivity factor mainly influences the early time, after which gas flow rates from the natural fracture decrease and can flow into the wellbore quickly - the hydraulic fracture equivalent for the infinity-conductivity fracture.

5 . The solution of this paper can solve the problem of historical matching, in which the matching speed was fast and the result was good.

Acknowledgments. This article was supported by National Science and Technology Major Project of China (Project No. 2016ZX05037003-002).

\section{References}

Albinali A., Holy R., Sarak H., Ozkan E. (2016) Modeling of 1D anomalous diffusion in fractured nanoporous media, Oil Gas Sci. Technol. - Rev. IFP Energies nouvelles 71, 56.

Aminian K., Ameri S. (2009) Predicting production performance of CBM reservoirs, J. Nat. Gas Sci. Eng. 1, 1, 25-30.
Anbarci K., Ertekin T. (1990) A comprehensive study of pressure transient analysis with sorption phenomena for single-phase gas flow in coal seams, SPE Annual Technical Conference and Exhibition of the Society of Petroleum Engineers, New Orleans, Louisiana, September 23-26.

Anbarci K., Ertekin T. (1992) Pressure transient behavior of fractured wells in coalbed reservoirs, SPE Annual Technical Conference and Exhibition, Washington, D.C., October 4-7.

Cinco-Ley H., Meng H.Z. (1988) Pressure transient analysis of wells with finite conductivity vertical fractures in double porosity reservoirs, SPE Annual Technical Conference and Exhibition, Houston, Texas, October 2-5.

Cinco-Ley H., Samaniego V.F., Dominguez A.N. (1978) Transient pressure behavior for a well with a finite-conductivity vertical fracture, SPEJ 18, 04, 253-264.

Clarkson C.R., Jordan C.L., Ilk D., et al. (2009) Production data analysis of fractured and horizontal CBM wells, SPE Eastern Regional Meeting, Charleston, West Virginia, September 23-25.

de Swaan A. (1976) Analytic solutions for determining naturally fractured reservoir properties by well testing, SPEJ 16, 03, 117-122.

de Swaan A. (2016) Pressure transients in a fractal-cluster model of porous media, Oil Gas Sci. Technol. - Rev. IFP Energies nouvelles $\mathbf{7 1}, 9$.

Gringarten A.C., Ramey H.J. Jr, Raghavan R. (1975) Applied pressure analysis for fractured wells, J. Pet. Technol. 27, 7, 887-892.

Kazemi H., Gilman V.J., Elsharkawy A.M. (1992) Analytical and numerical solution of oil recovery from fractured reservoirs with empirical transfer functions, SPEJ 7, 02, 219-227.

King G.R., Ertekin T., Schwerer F.C. (1986) Numerical simulation of the transient behavior of coal-seam degasification wells, SPE Form. Eval. 1, 02, 165-183.

Li X., Zhang D., Li S. (2015) A multi-continuum multiple flow mechanism simulator for unconventional oil and gas recovery, J. Nat. Gas Sci. Eng. 26, 652-669.

Mcguire W.J., Sikora V.J. (1960) The effect of vertical fractures on well productivity, J Pet Technol. 12, 10, 72-74.

Meunier D.F., Kabir C.S., Wittmann M.J. (1984) Gas well test analysis: use of normalized pressure and time functions, SPE Annual Technical Conference and Exhibition, Houston, Texas, September 16-19.

Nie R.S., Meng Y.F., Guo J.C., et al. (2012) Modeling transient flow behavior of a horizontal well in a coal seam, Int. J. Coal Geol. 922, 54-68.

Ozkan E., Raghavan R. (1988) Some new solutions to solve problems in well test analysis problems: part 1 - analytical considerations, SPE Form. Eval. 6, 3, 359-368.

Ozkan E., Raghavan R. (1991) New solutions for well-testanalysis problems: part 2 - computational considerations and applications, SPE Form. Eval. 6, 3, 369-378.

Raghavan R., Cady G.V., Ramey H.J. (1972) Well test analysis for vertically fractured wells, J. Pet. Technol. 24, 8, 1014-1020.

Riley M.F., Brigham W.E., Horne R.N. (1991) Analytic solutions for elliptical finite-conductivity fractures, SPE Annual Technical Conference and Exhibition, Dallas, Texas, October 6-9.

Wang X., Luo W., Hou X., et al. (2014) Transient pressure analysis of multiple-fractured horizontal wells in boxed reservoirs, Petrol. Explor. Dev. 41, 1, 74-78.

Warren J.E., Root P.J. (1962) The behavior of naturally fractured reservoirs, SPEJ 3, 3, 245-255. 